\title{
Composición y superposición de dietas en cuatro especies de Diplectrum (Perciformes: Serranidae) en el Pacífico central mexicano
}

\author{
Bernabé Aguilar Palomino \& Gaspar González Sansón \\ Departamento de estudios para el desarrollo sustentable de zonas costeras, Universidad de Guadalajara, México, \\ Gómez Farias 82, San Patricio-Melaque, Jalisco, México. CP. 48980; baguilar007@yahoo.com, \\ gaspargonzalez2001@yahoo.es
}

Recibido 21-IX-2009. Corregido 03-V-2010. Aceptado 10-VI-2010.

\begin{abstract}
Analysis of diet composition and overlap in four species of the genus Diplectrum (Perciformes: Serranidae) in the Mexican Central Pacific. The information of trophic interactions among species is essential to understand ecosystem function. To assess this in four Diplectrum species, we analyzed the stomach contents of 397 individuals caught using shrimp trawling nets off the coasts of Jalisco and Colima, Mexico. Main food component of D. eumelum were fish of the Order Pleuronectiformes, followed by shrimps (Metapenaeopsis spp). D. euryplectrum feeds mainly on crustaceans, with stomatopods (Squilla mantoidea) as main food items followed by shrimps and brachyurans. Fish and polychaetes had a low representation in the diet of this species. D. labarum feeds mainly on crustaceans, mollusks and fish. The stomatopod Eurysquilla veleronis contributed to with a high diet percentage by weight, followed by shrimps (mostly Solenocera florae) and brachyurans, as well as the squid Lolliguncula diomedae. Fishes (Ophidion spp.) were also an important component of the diet of this species. The most common preys of D. rostrum were crustaceans, mollusks, polychaetes and fish. The squid Lolliguncula diomedae and fishes of genus Ophidion were also well represented in the diet. Diet breadth index yielded significant differences between species. The number of diet items varied from 16 in D. euryplectrum to 6 in $D$. eumelum. The average value of overlap $(0.247)$ was not significantly different $(\mathrm{p}=0.118)$ from the expected one for a null model (0.174). The observed variance of the overlap index (0.071) was significantly higher $(\mathrm{V}=0.025, \mathrm{p}=0.0004)$ than the value expected for a null model. Rev. Biol. Trop. 58 (4): 1189-1198. Epub 2010 December 01.
\end{abstract}

Key words: Diplectrum, diet, trophic interactions, overlaps, central Pacific.

Comprender las interacciones tróficas en un ecosistema resulta esencial para entender la dinámica del mismo (Barrera-Oro 2003, Hajisamae 2009). La competencia por los recursos alimentarios se acepta comúnmente como un mecanismo básico en la conformación de las comunidades marinas en general y de la ictiofauna en particular (Platell \& Potter 2001, Raborn et al. 2004). No obstante, resulta muy difícil en la práctica poder verificar la existencia real de competencia por el alimento, debido a que un número grande de factores también influyen en la conformación de las comunidades, generalmente no se conocen en detalle y su efecto combinado oscurece notablemente el posible resultado de las interacciones tróficas (Raborn et al. 2004).

Es importante resaltar, sin embargo, que para poder comenzar a comprender los posibles mecanismos de competencia, es imprescindible conocer bien la composición y superposición de las dietas de las especies (Gotelli \& Graves 1996).

Interés particular tiene la investigación de la dieta de especies que coexisten en un mismo hábitat y presentan una morfología general muy similar. Tal es el caso de las especies del género Diplectrum que habitan en las aguas 
costeras del Océano Pacífico central mexicano (POT), especialmente en las costas de Jalisco y Colima. Para esta región han sido consignadas cuatro especies del género mencionado (Aguilar-Palomino et al. 1996, 2001). Prácticamente todas las referencias sobre estas especies en el Pacífico, remiten invariablemente a los trabajos de Bortone (1977c), Fischer et al. (1995), Gómez et al. (1999) y López-Peralta \& Arcila (2002). A partir de estos trabajos, se puede resumir que éstas son especies bentónicas, que habitan en fondos fangosos o arenosos, son de tamaño pequeño (menores de $30 \mathrm{~cm}$ de longitud total) y muy similares en su morfología externa (Gibran 2007), según López et al. (2002), el género Diplectrum es endémico de América y sus especies son hermafroditas sincrónicas, pero existe muy poca información sobre la biología de estas especies.

Varios autores han trabajado sobre los aspectos reproductivos de especies del género (Bortone 1977a, 1977b, Obando \& León 1989, Tuart \& Bortone 2006). Ruíz-Ramírez et al. (1997) publicaron información sobre la relación largo-peso de estas especies. Sobre los hábitos alimentarios de las especies de Diplectrum, se conoce el trabajo de Gibran (2007) para las costas del Océano Atlántico. En el Pacífico oriental tropical se conocen los trabajos de Bortone (1977a), Gómez et al. (1999), López-Peralta \& Arcila 2002 y Cruz-Corona (2004).

El objetivo de nuestra investigación fue realizar un análisis comparativo de las dietas de las cuatro especies del género Diplectrum que son frecuentes en la región del POT. La hipótesis de trabajo fue que existen diferencias significativas en sus dietas, lo cual ayuda a explicar la coexistencia de especies con morfología muy similar en un mismo hábitat.

\section{MATERIAL Y MÉTODOS}

Área de estudio y estaciones de muestreo: El área de estudio está definida como la franja de la plataforma continental que se extiende desde la desembocadura del río Cuitzmala en el estado de Jalisco, México (19 $21^{\prime}$ N $-105^{\circ} 01^{\prime}$ W) al norte y hacia el sur hasta el área marina frente al poblado de Cuyutlán, en el estado de Colima $\left(18^{\circ} 55^{\prime} \mathrm{N}-104^{\circ} 07^{\prime} \mathrm{W}\right)$. En esta área que tiene una longitud de $100 \mathrm{~km}$, se ubicaron siete estaciones de muestreo (Cuadro 1).

La línea de costa del área estudiada es irregular y presenta tres accidentes importantes, constituidos por la Bahía de Tenacatita, Bahía de Navidad y el grupo de Bahías ManzanilloSantiago; el resto de la línea costera del área está formada por playas relativamente extensas y poco protegidas. Asimismo, la zona de estudio presenta sedimentos blandos, integrados fundamentalmente por limos arenosos que contienen cantidades variables de materia orgánica (Aguilar-Palomino et al. 1996). Los patrones de corrientes en la zona se pueden inferir a partir de las pautas típicas de circulación para el Océano Pacífico oriental (Pacheco 1991, Filonov et al. 2000, Kessler 2006).

CUADRO 1

Estaciones de muestreo (coordenadas son aproximadamente las del centro geográfico de la localidad)

TABLE 1

Sampling stations (coordinates are at the approximated center of the locality)

\begin{tabular}{cll} 
No. & \multicolumn{1}{c}{ Descripción } & \multicolumn{1}{c}{ Latitud, Longitud } \\
\hline 1 & Plataforma continental frente a la desembocadura del Río Cuitzmala & $19^{\circ} 21^{\prime} \mathrm{N}, 105^{\circ} 01^{\prime} \mathrm{W}$ \\
2 & Bahía de Tenacatita & $19^{\circ} 16^{\prime} \mathrm{N}, 104^{\circ} 50^{\prime} \mathrm{W}$ \\
3 & Bahía de Navidad & $19^{\circ} 10^{\prime} \mathrm{N}, 104^{\circ} 43^{\prime} \mathrm{W}$ \\
4 & Plataforma continental frente a Playa El Coco & $19^{\circ} 09^{\prime} \mathrm{N}, 104^{\circ} 40^{\prime} \mathrm{W}$ \\
5 & Bahías de Cenicero, Santiago y Manzanillo & $19^{\circ} 04^{\prime} \mathrm{N}, 104^{\circ} 25^{\prime} \mathrm{W}$ \\
6 & Plataforma continental frente a la Laguna Cuyutlán, boca de Tepalcates & $18^{\circ} 58^{\prime} \mathrm{N}, 104^{\circ} 14^{\prime} \mathrm{W}$ \\
7 & Plataforma continental frente a la Laguna Cuyutlán, cerca de Cuyutlán & $18^{\circ} 55^{\prime} \mathrm{N}, 104^{\circ} 07^{\prime} \mathrm{W}$
\end{tabular}


Obtención y procesamiento de las muestras: En primavera y verano de 1995 e invierno de 1996 se capturaron ejemplares de Diplectrum eumelum, D. euryplectrum, D. labarum y $D$. rostrum. Las capturas se realizaron utilizando el barco camaronero "BIP V" del Departamento de estudios para el Desarrollo Sustentable de la Zona Costera de la Universidad de Guadalajara. Se utilizaron redes camaroneras tipo semi-portuguesa, con una abertura de trabajo calculada en la boca de $6.9 \mathrm{~m}$, una altura calculada entre las relingas de $1.15 \mathrm{~m}$ y un tamaño de malla estirada en el copo de $38 \mathrm{~mm}$.

En cada estación de muestreo se realizaron cuatro arrastres a profundidades de 20, 40,60 y $80 \mathrm{~m}$. Cada arrastre tuvo $30 \mathrm{~min}$ de duración y se realizó con dos redes simultáneamente y a una velocidad media de $3.6 \mathrm{~km} /$ hora (González-Sansón et al. 1997). Los peces fueron introducidos en bolsas de polietileno debidamente rotuladas indicando el nombre de la especie, la fecha de recolección y el número de la muestra, procediendo a congelarlos a $-10^{\circ} \mathrm{C}$ para su preservación. En el momento del procesamiento, se extrajeron los estómagos y se colocaron en cajas de Pétri para su análisis con la ayuda de un microscopio estereoscópico. Todas las entidades alimentarias fueron identificadas hasta el nivel taxonómico más bajo posible, dependiendo del estado de digestión en que se encontraban. Para el caso de los peces presas completos en un estado de digestión mínima, la determinación taxonómica se realizó mediante claves generales, utilizando los trabajos de Allen \& Robertson (1994) y Fischer et al. (1995). La identificación taxonómica de los restos de peces se baso en el conteo de vértebras y se realizó empleando los trabajos de Clothier (1950). La determinación taxonómica de crustáceos se basó en sus apéndices anatómicos, utilizando las claves de Fischer et al. (1995). Los poliquetos fueron identificados con los trabajos de Brusca (1980) y Salazar-Vallejo et al. (1988). En el caso de los moluscos se emplearon los trabajos de Keen (1971) y Fischer et al. (1995).

El número de estómagos necesarios para obtener una representación adecuada del contenido estomacal se verificó utilizando el método de curvas acumuladas del número de entidades alimentarias al incrementar el número de estómagos (Laptyhovsky 2005). En todas las especies el número de estómagos examinados rebasó ampliamente el tamaño de muestra mínimo necesario para alcanzar el valor asintótico del número de entidades alimenticias. La composición de la dieta fue expresada según el porcentaje en número $(\mathrm{N})$ y en peso $(\mathrm{P})$, así como la frecuencia de aparición (F) de acuerdo con Hyslop (1980). Para sintetizar en un solo índice la importancia general de las entidades alimentarias se utilizó el índice de importancia relativa (IIR) descrito por Pinkas et al. (1971) cuya fórmula es: $\mathrm{IIR}=(\mathrm{N}+\mathrm{P}) \mathrm{F}$. Este índice fue expresado también en porcentaje para cada entidad alimentaria.

Análisis cuantitativo: Para estimar la amplitud de las dietas se utilizaron el índice de Smith (basado en el porcentaje de peso), el número de categorías alimentarias (presas) observadas y el número de presas más frecuentes expresado como una fracción del número total de categorías alimentarias. Estos indicadores se describen en detalle en Krebs (1998). La amplitud fue calculada utilizando el sistema "Programs for Ecological Methodology, 2 ed." Versión 6.1.1 (2003).

Para estimar la superposición de las dietas se utilizó el índice de Levins modificado por Pianka (1973), según se describe detalladamente en Krebs (1998). El cálculo de los índices y las simulaciones para determinar diferencias significativas con el modelo nulo correspondiente se hicieron utilizando el sistema "EcoSim" Versión 7.00 (Gotelli \& Entsminger 2001) y las reglas de análisis descritas en el mismo. Las simulaciones se basaron en 1000 iteraciones, considerando los estados de recurso igualmente probables y el algoritmo de aleatoriedad tres, que implica retener las amplitudes de dieta originales, con los estados cero no retenidos. La representación gráfica de la matriz de índices de superposición entre todos los pares de especies se realizó mediante escalado multidimensional no 
métrico utilizando el programa STATISTICA $6.0^{\circledR}$ (Stasoft).

\section{RESULTADOS}

Composición de las dietas: Se analizaron 129 ejemplares de Diplectrum eumelum. De estos, 45 individuos de tallas entre 5.7 y $17.9 \mathrm{~cm}$ de longitud total presentaron contenido estomacal (Cuadro 2). Se identificaron seis categorías alimentarias pertenecientes a crustáceos y peces. De acuerdo al IIR los crustáceos registraron dominancia de camarones del género Metapenaeopsis con pequeñas cantidades de estomatópodos y otros camarones. Los peces formaron la mayor parte de la dieta y las especies identificadas pertenecientes en todos los casos al orden Pleuronectiformes.

Se analizaron 236 ejemplares de Diplectrum euryplectrum. De estos, 152 individuos de tallas entre 4.1 y $16.2 \mathrm{~cm}$ de longitud total tenían contenido estomacal (Cuadro 2). Se identificaron 16 categorías alimentarias pertenecientes a crustáceos, anélidos y peces. En los crustáceos dominaron ampliamente los estomatópodos, grupo en el que la especie Squilla mantoidea estuvo representada en un alto porcentaje. El resto fueron camarones y braquiuros. Los peces y poliquetos tuvieron una representación muy baja en la dieta.

Se analizaron 431 ejemplares de Diplectrum labarum. De estos, 101 individuos de tallas entre 8.1 y $22.6 \mathrm{~cm}$ de longitud total tenían contenido (Cuadro 2). Se identificaron 14 categorías alimentarias pertenecientes a crustáceos, moluscos y peces. En los crustáceos dominaron ampliamente los estomatópodos, grupo en el que la especie Eurysquilla veleronis estuvo representada en un alto porcentaje. El resto fueron camarones (principalmente Solenocera florea) y braquiuros. El calamar dardo Lolliguncula diomedae tuvo una representación significativa en términos de peso, aunque presentó una baja incidencia numérica y frecuencia de aparición. El grupo de los peces constituyó también un componente importante de la dieta y estuvo formado principalmente por ejemplares pequeños del género Ophidion.
Se analizaron 790 ejemplares de Diplectrum rostrum. De estos, 99 individuos de tallas entre 6.5 y $28.1 \mathrm{~cm}$ de longitud total presentaron contenido estomacal (Cuadro 2). Se identificaron 13 categorías alimentarias pertenecientes a crustáceos, moluscos, poliquetos y peces. En el caso de los crustáceos, se identificaron braquiuros, camarones y estomatópodos, pero no se encontró una entidad dominante. El calamar dardo Lolliguncula diomedae tuvo una representación importante en términos de peso, aunque presentó una baja incidencia numérica y frecuencia de aparición. Los peces fueron un componente importante de la dieta, destacándose los ejemplares pequeños del género Ophidion.

Algunas categorías alimentarias presentaron diferencias notables en cuanto a su importancia según el método cuantitativo usado (numérico, gravimétrico, de frecuencia o índice de importancia relativa). No obstante, un análisis general de correlación permitió comprobar que en general, todos los métodos tienden a dar a las categorías un orden de importancia similar. Para ese análisis, se combinaron todos los cuartetos de valores de importancia calculados según los cuatro métodos en las especies investigadas y se calcularon los coeficientes de correlación por rangos de Spearman entre las series obtenidas $(n=49)$. Todos los valores de correlación oscilaron entre 0.66 y 0.90 , resultando significativos $(\mathrm{p}<0.05)$ en todos los casos.

Amplitud de las dietas: El valor del índice de Smith varió entre 0.79 en Diplectrum euriplectrum y 0.92 en Diplectrum eumelum. La superposición o no de los intervalos de confianza del índice fueron utilizados como criterio para definir la existencia de diferencias significativas entre pares de especies (Fig. 1). Según este criterio, D. eumelum presentó una amplitud de dieta superior al resto de las especies (el límite inferior de su intervalo de confianza es mayor que el superior de ese mismo intervalo en el resto de las especies). Este índice se puede considerar significativamente mayor en $D$. rostrum con respecto a $D$. euryplectrum 
CUADRO 2

Composición de la dieta de cuatro especies de Diplectrum capturadas en las aguas costeras de Jalisco y Colima, México

TABLE 2

Diet composition of four species of Diplectrum caught in coastal waters of Jalisco and Colima, Mexico

\begin{tabular}{|c|c|c|c|c|c|c|c|c|c|c|c|c|c|c|c|c|}
\hline \multirow{2}{*}{ Presa } & \multicolumn{4}{|c|}{ Diplectrum eumelum } & \multicolumn{4}{|c|}{ D. euryplectrum } & \multicolumn{4}{|c|}{ Diplectrum labarum } & \multicolumn{4}{|c|}{ Diplectrum rostrum } \\
\hline & $\mathrm{N}$ & $\mathrm{G}$ & FO & IIR & $\mathrm{N}$ & $\mathrm{G}$ & FO & IIR & $\mathrm{N}$ & $\mathrm{G}$ & FO & IIR & $\mathrm{N}$ & $\mathrm{G}$ & FO & IIR \\
\hline \multicolumn{17}{|l|}{ Crustáceos } \\
\hline Squilla mantoidea & 35 & 5.9 & 11.4 & 7.1 & 14 & 41 & 29.1 & 61.2 & 13.9 & 5.2 & 14.1 & 10.1 & 10.8 & 7.8 & 15.8 & 12 \\
\hline Squilla spp. & & & & & 2.7 & 11 & 12.6 & 6.1 & 5.6 & 2.7 & 0.8 & 2 & & & & \\
\hline Eurysquilla veleronis & & & & & 4 & 18 & 16.4 & 13.4 & 25.8 & 17 & 26.5 & 41.3 & 1.8 & 1.4 & 2.6 & 0.3 \\
\hline Eurysquilla spp. & & & & & 0.4 & 3.8 & 0.6 & 0.2 & 1.9 & 0.2 & 7.4 & 0.2 & & & & \\
\hline Metapenaeopsis mineri & & & & & 0.2 & 0.3 & 1 & 0 & & & & & 12.6 & 6.5 & 15.7 & 12.3 \\
\hline Metapenaopsis spp. & 13 & 19 & 17.3 & 16.6 & & & & & 4.6 & 2.4 & 5.2 & 1.4 & & & & \\
\hline Euphylax spp. & & & & & 0.8 & 1 & 1.9 & 0.1 & & & & & 1.8 & 1.1 & 1.6 & 0.3 \\
\hline Trachypenaeus brevisuturae & 4.4 & 6.9 & 10.4 & 1.9 & 0.2 & 3.7 & 3 & 0.1 & & & & & 2.7 & 3.1 & 3.9 & 0.9 \\
\hline Euphylax dovii & & & & & 0.9 & 1.5 & 6.8 & 0.4 & 0.9 & 0.1 & 1.2 & 0.1 & 16.3 & 6.8 & 11.8 & 11.1 \\
\hline Gecarcinus quadratus & & & & & 0.6 & 1 & 2.8 & 0.2 & & & & & & & & \\
\hline Solenocera florea & & & & & 1.8 & 5.9 & 11 & 2.5 & 11.1 & 8.7 & 7.9 & 6.4 & & & & \\
\hline Solenocera spp. & & & & & 0.2 & 1 & 0.8 & 0.1 & & & & & & & & \\
\hline Portunus xantusii xantusii & & & & & & & & & 3.7 & 2.3 & 4.9 & 1 & 24.3 & 6.2 & 10.5 & 13.1 \\
\hline Portunus xantusii affinis & & & & & & & & & 3.7 & 1.6 & 2.5 & 0.4 & & & & \\
\hline Portunus xantusii & & & & & & & & & 1.9 & 1.8 & 2.5 & 0.3 & & & & \\
\hline Portunus spp. & & & & & & & & & 1.9 & 0.7 & 2.5 & 0.2 & & & & \\
\hline Cumaceo & & & & & 48 & 2.5 & 5.7 & 10.7 & & & & & & & & \\
\hline Hepatus lineatus & & & & & & & & & & & & & 0.9 & 0.3 & 1.3 & 0.1 \\
\hline Protachypene precipua & & & & & & & & & & & & & 8.1 & 3.4 & 9.2 & 4.3 \\
\hline Larvas de camarón & & & & & 25 & 6 & 3.4 & 4.4 & & & & & & & & \\
\hline \multicolumn{17}{|l|}{ Peces } \\
\hline Pleuronectiformes & 13 & 26 & 17.3 & 20.2 & & & & & & & & & & & & \\
\hline Symphurus spp. & 20 & 23 & 24 & 33 & 0.2 & 0.3 & 1 & 0.1 & & & & & & & & \\
\hline Bothus spp. & 15 & 20 & 19.6 & 21.2 & & & & & & & & & & & & \\
\hline Ophidion spp. & & & & & 0.8 & 2.8 & 2.9 & 0.4 & 21.3 & 22 & 20.5 & 33.5 & 15.3 & 32.4 & 19.8 & 40.7 \\
\hline Synodus lacertinus & & & & & & & & & & & & & 1.8 & 14.1 & 2.6 & 1.7 \\
\hline Huevos de peces & & & & & & & & & 1.9 & 4.1 & 1.5 & 0.5 & & & & \\
\hline Poliquetos & & & & & 0.2 & 0.5 & 1 & 0.1 & & & & & & & & \\
\hline Crysopetalum spp. & & & & & & & & & & & & & 0.9 & 0.3 & 1.3 & 0.1 \\
\hline \multicolumn{17}{|l|}{ Moluscos } \\
\hline Lolliguncula diomedae & & & & & & & & & 1.8 & 31 & 2.5 & 2.6 & 2.7 & 16.6 & 3.9 & 3.1 \\
\hline
\end{tabular}

$\mathbf{N}=$ Porcentaje en número; $\mathbf{G}=$ Porcentaje en peso; $\mathbf{F O}=$ Porcentaje de frecuencia de ocurrencia; $\mathbf{I I R}=$ Índice de importancia relativa expresado en porcentaje.

$\mathbf{N}=$ Percentage in number; $\mathbf{G}=$ Percentage in weight; $\mathbf{F O}=$ Frequency of occurrence as percentage; IIR $=$ Index of relative importance as percentage. 


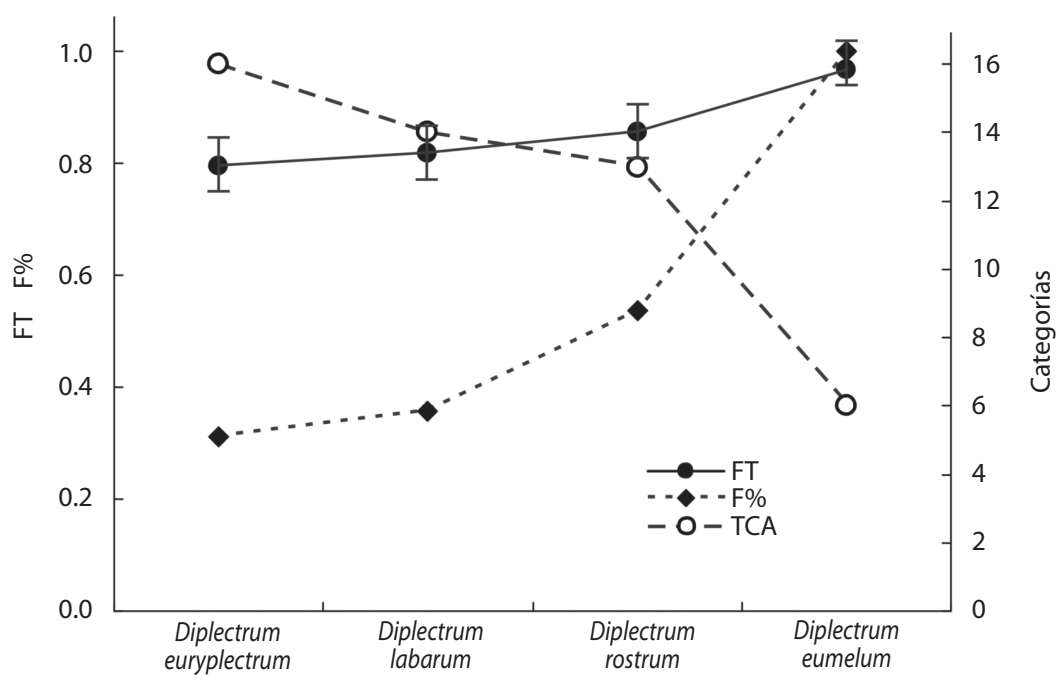

Fig. 1. Índice de Smith (FT, con intervalos de confianza del 95\%) para las categorías alimentarias usadas más frecuentemente (F\%) y total de categorías alimentarias (TCA) para las cuatro especies.

Fig. 1. Smith's Index values (FT, with 95\% confidence intervals), most frequently used food items (F\%) and total food items (TCA) for the four species.

porque el intervalo de confianza de la primera especie no incluye el valor calculado del índice de la segunda y viceversa.

La proporción de categorías alimentarias utilizadas con más frecuencia tuvo un comportamiento similar al índice de Smith, pero con cambios más notables e incrementó desde 0.31 en D. euryplectrum hasta 1.00 en D. eumelum. Por el contrario, el número total de categorías alimentarias identificadas en cada especie, presentó una tendencia inversa con respecto a los dos índices ya mencionados y disminuyó desde 16 en $D$. euryplectrum hasta seis en $D$. eumelum.

Superposición de las dietas: $D$. eumelum, con una dieta a base de peces y decápodos principalmente, presentó un superposición muy baja (0.02-0.13) con respecto al resto de las especies, mientras que D. labarum y D. rostrum registraron un superposición relativamente alta (0.74) entre sí (consumo de peces y calamares) y moderado $(0.24,0.33)$ con respecto a $D$. euryplectrum, cuya dieta se basa fundamentalmente en estomatópodos y decápodos. El escalado multidimensional permitió representar de forma objetiva la relación de la superposición entre todas las especies simultáneamente y visualizar claramente las diferencias esenciales en el espectro trófico de las especies (Fig. 2). En este caso, por tratarse de sólo cuatro especies a ordenar, el diagrama hizo una representación exacta y la medida de la falta de ajuste, conocida en inglés como "stress" fue igual a cero.

El valor medio de la superposición (0.247) no fue significativamente diferente $(\mathrm{p}=0.118)$ al esperado para un modelo nulo (0.174). La varianza observada de los índices de superposición (0.071) fue significativamente mayor $(\mathrm{V}=0.025, \mathrm{p}=0.0004)$ al valor medio obtenido en la simulación basada en un modelo nulo.

\section{DISCUSIÓN}

Como han sugerido Beyst et al. (1999), si la composición y superposición de las dietas 


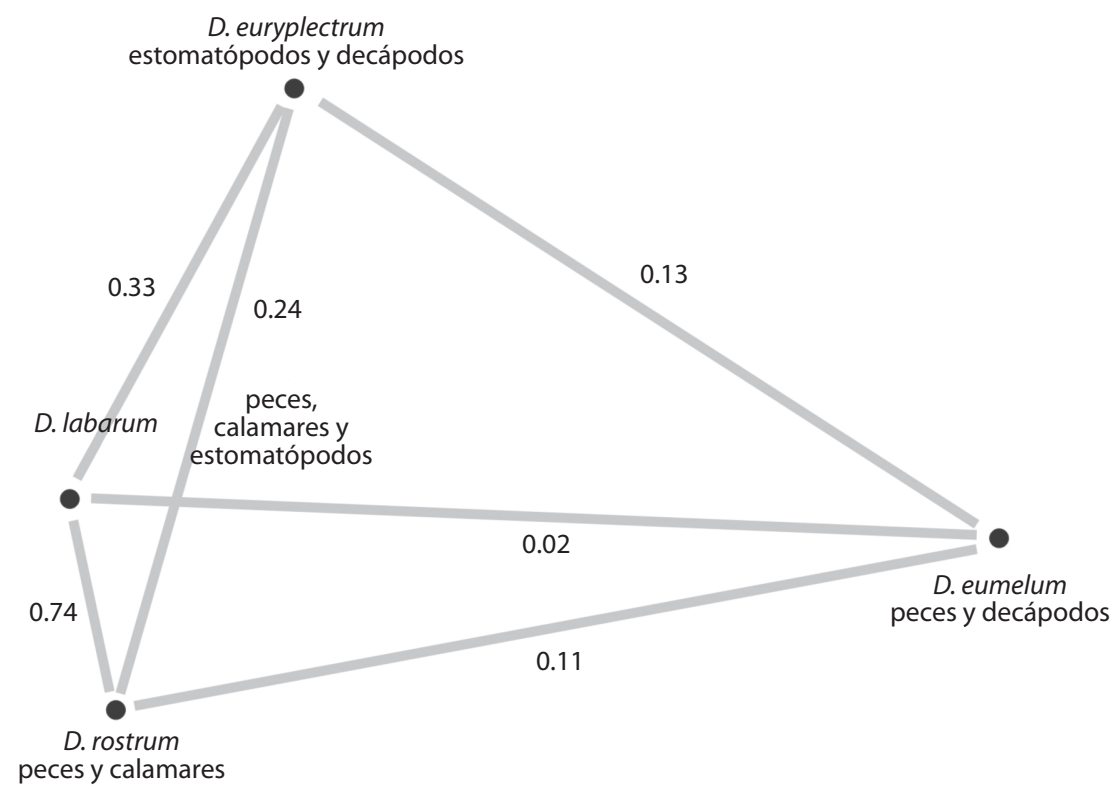

Fig. 2. Representación por MDS de la sobreposición de las dietas entre las cuatro especies. Los números indican el valor del índice de Pianka para cada par de especies. Se indican las categorías alimentarias más importantes.

Fig. 2. MDS representations of diet overlap between the four species. Numbers indicate the value of Pianka's index for each pair of species. Most important food items indicated (calamares=squids, decápodos=decapods, estomatópodos=stomatopods, peces $=$ fish).

se basa en recursos alimentarios muy abundantes, entonces lo más probable es que esté ocurriendo un aprovechamiento oportunista de dichos recursos, conducta observada por Gibran (2007) que considera a Diplectrum formosum y $D$. radiale como depredadores visuales oportunistas. En estas condiciones debe suponerse que la competencia por alimento es baja y las diferencias observadas entre especies podrían deberse a otros factores relacionados con aspectos sutiles de la morfología y la conducta de las mismas. Este enfoque concuerda con lo observado por Gibran (2007), quién señala que las características morfológicas de las especies de Diplectrum, se asocian con el uso de recursos tróficos determinados. Los componentes tróficos registrados para especies de Diplectrum por Ferreira \& Abiloha (2005) y Gibran (2007) en las costas del Océano Atlántico, así como los observados en el Pacífico oriental tropical por otros autores (Bortone 1977a, Gómez et al. 1999, López-Peralta \& Arcilla 2002, Cruz-Corona 2004), coinciden con la presencia de macroinvertebrados y peces en los espectros tróficos. Las dietas de estas especies, están compuestas en orden de importancia, por crustáceos, peces y moluscos. Esta información coincide con lo encontrado en el presente trabajo. Godínez-Domínguez \& González-Sansón (1999) ofrecen datos sobre la composición de las comunidades de invertebrados presentes en la misma época y zona donde se capturaron los individuos de nuestro estudio. Esa información se obtuvo en los mismos arrastres en los cuales se recolectaron las muestras para nuestro análisis del contenido estomacal. En términos generales se puede apreciar que las categorías alimentarias que aparecen con mayor abundancia en los estómagos analizados, coinciden con los grupos 
que conforman la mayoría de la comunidad de invertebrados (jaibas, cangrejos, camarones y estomatópodos) presentes en la zona. No obstante, una comparación cuantitativa no es posible, pues las especies de Diplectrum consumen los mismos grupos consignados en GodínezDomínguez \& González-Sansón (1999) pero seleccionan tallas menores. De hecho, se puede considerar que estas especies se alimentan de jóvenes de otras especies principalmente y tal vez de camarones adultos pero de tamaño pequeño.

El valor medio de superposición no significativo obtenido en este estudio se puede considerar como una evidencia de que no existe una competencia fuerte por los recursos en las especies estudiadas. Gotelli \& Graves (1996) afirman que niveles significativamente bajos de índice medio de superposición pueden indicar una competencia efectiva y una repartición de los recursos alimentarios, mientras que valores significativamente altos indican una utilización compartida de los recursos y falta de competencia por los mismos. Estos mismos autores reconocen que es posible que un nivel de superposición alto pueda implicar una competencia muy fuerte que aún no ha producido una divergencia en el uso de los recursos. Pero en cualquier caso, es muy difícil sacar una conclusión firme sobre la existencia o no de competencia por alimento sin tener más información sobre la disponibilidad de recursos y otros aspectos relacionados con la interacción entre especies.

Según Winemiller \& Pianka (1990), una varianza significativamente alta de valores de superposición, como la obtenida en esta investigación, puede indicar cierta estructura en gremios. En este contexto, algunos pares de especies son muy similares en el uso que hacen de los recursos alimentarios, tal como D. rostrum y D. laborum, mientras que otras son muy diferentes con respecto al resto, como por ejemplo $D$. eumelum. Esto no implica que exista competencia por recursos, ya que estas diferencias y similitudes se pueden deber a la disponibilidad y abundancia de recursos (Bradley \& Bradley 1985) o estar condicionadas por adaptaciones morfológicas y conductuales que no han sido identificadas apropiadamente.

\section{RESUMEN}

Para comprender las interacciones tróficas en un ecosistema, es imprescindible conocer bien la composición y superposición de las dietas de las especies que coexisten en un mismo hábitat y presentan una morfología general muy similar. Se analizaron los contenidos estomacales de 397 ejemplares de cuatro especies de Diplectrum, capturados con redes camaroneras frente a las costas de Jalisco y Colima, México. D. eumelum consumió principalmente peces del orden Pleuronectiformes, seguido por camarones del género Metapenaeopsis y estomatópodos. D. euryplectrum registró en su contenido estomacal, estomatópodos (Squilla mantoidea), seguido por camarones y braquiuros. D. labarum consumió crustáceos, moluscos y peces. El estomatópodo Eurysquilla veleronis registró un alto porcentaje, seguido por camarones (principalmente Solenocera florea) y braquiuros, así como el calamar dardo Lolliguncula diomedae. Los peces (género Ophidion principalmente) constituyeron un componente importante de la dieta de esta especie. En la dieta de D. rostrum se identificaron crustáceos, moluscos, poliquetos y peces. El calamar dardo Lolliguncula diomedae y los peces del género Ophidion tuvieron una alta representación. Se observaron diferencias significativas entre los índices de amplitud de dietas de las especies analizadas. El número de entidades consumidas disminuyó desde 16 en $D$. euryplectrum hasta seis en $D$. eumelum. El valor medio de superposición (0.247) no resultó significativamente diferente $(\mathrm{p}=0.118)$ del esperado para un modelo nulo (0.174). La varianza observada de los índices de superposición (0.071) fue significativamente mayor $(\mathrm{V}=0.025, \mathrm{p}=0.0004)$ que el valor medio obtenido en la simulación basada en un modelo nulo.

Palabras clave: Diplectrum, dieta, superposición, Pacífico central.

\section{REFERENCIAS}

Aguilar-Palomino, B., J. Mariscal-Romero, G. GonzálezSansón \& L.E. Rodríguez-Ibarra. 1996. Ictiofauna demersal de fondos blandos de la plataforma continental de Jalisco y Colima, México, en la primavera de 1995. Cienc. Mar. 22: 469-481.

Aguilar-Palomino, B., C. Pérez-Reyes, F. Galván-Magaña \& L.A. Abítia-Cárdenas. 2001. Ictiofauna de la Bahía de Navidad, Jalisco, México. Rev. Biol. Trop. 49: 173-190.

Allen, G.R. \& R.D. Robertson. 1994. Fishes of the Tropical Eastern Pacific. Universidad de Hawaii, Hawaii, EEUU. 
Barrera-Oro, E. 2003. Analysis of dietary overlap in Antarctic fish (Notothenioidei) from the South Shetland Islands: no evidence of food competition. Polar Biol. 26: 631-637.

Beyst, B., A. Cattrijsse \& J. Mees. 1999. Feeding ecology of juvenile flatfishes of the surf zone of a sandy beach. J. Fish Biol. 55: 1171-1186.

Bortone, S.A. 1977a. Observations of the life history of Diplectrum pacificum and D. macropoma (Pisces, Serranidae) from the Bay Panama. Pac. Sci. 1: $49-60$.

Bortone, S.A. 1977b. Gonad morphology of the hermaphroditic fish Diplectrum pacificum (Serranidae). Copeia 448-453.

Bortone, S.A. 1977c. Revision of the sea basses of the genus Diplectrum (Pisces: Serranidae) (NOAA tech. Rep. NMFS circular-404).

Bradley, R.A \& D.W. Bradley. 1985. Do non-random patterns of species in niche space imply competition? Oikos 45: 443-445.

Brusca, R.C. 1980. Common intertidal invertebrates of the Gulf of California. Universidad de Arizona, Arizona, EEUU.

Clothier, C.R. 1950. A key to some southern California fishes based on vertebral characters. California Department of Fishing Game. Fish Bull. 79.

Cruz-Corona, J.L. 2004. Componente trófico del género Diplectrum: Serranidae (Perciformes) en las costas de Jalisco y Colima, México. Tesis de Licenciatura, Instituto Tecnológico del Mar, Nayarit, México.

Ferreira, C. \& V. Abiloha. 2005. A alimentacao da michole Diplectrum radiale (Quoy \& Gaimard, 1824) em um banco areno-lodoso na ilha do mel, Parana, Brasil. Est. Biol. 27: 13-17.

Filonov, A.E., I.E. Tereschenko, C.O. Monzon, M.E. González-Ruelas \& E. Godínez-Domínguez. 2000. Variabilidad estacional de los campos de temperatura y salinidad en la zona costera de los estados de Jalisco y Colima, México. Cienc. Mar. 26: 303-321.

Fischer, W., F. Krupp, W. Schneider, C. Sommer, K.E. Carpenter \& V. Niem. 1995. Pacífico Centro-Oriental. Guía FAO para identificación de especies para los fines de la pesca. FAO, Roma, Italia.

Gibran, F.Z. 2007. Activity, habitat use, feeding behavior and diet of tour sympatric species of Serranidae (Actinopterigii: Perciformes) in southeastern Brasil. Neot. Ichthy. 5: 387-398
Godínez-Domínguez, E \& G. González-Sansón. 1999. Diversidad de macroinvertebrados de fondos blandos de la plataforma continental de Jalisco y Colima, México. Cien. Mar. 25: 609-627.

Gómez, C.G.O., L.A. Zapata, R. Franke \& G.E. Ramos. 1999. Hábitos alimentarios de Epinephelus acanthistius y notas de otros peces serranidos capturados en el parque nacional natural de Gorgona, Pacífico colombiano. Inv. Mar. Cost. 28: 43-60.

González-Sansón, G., B. Aguilar-Palomino, A.J. Arciniega-Flores, R. García de Quevedo, E. GodínezDomínguez, V. Landa, J. Mariscal-Romero \& J.E. Michel-Morfin. 1997. Variación espacial de la abundancia de la fauna de fondos blandos en la plataforma continental de Jalisco y Colima, (Primavera, 1995). Cien. Mar. 23: 93-110.

Gotelli, N.J. \& G.R. Graves. 1996. Null Models in Ecology. Smithsonian Institution, Washington D.C., EEUU.

Gotelli, N.J \& G.L. Entsminger. 2004. EcoSim: Null models software for ecology. Version 7.0. Acquired Intelligence Inc. \& Kesey-Bear. Jericho, VT 05465. (Consultado 8 junio 2010, http://garyentsminger.com/ ecosim/index.htm).

Hajisamae, S. 2009. Trophic ecology of bottom fishes assemblage along coastal areas of Thailand. Est. Coast. Shelf Sci. 82: 503-514.

Hyslop, E.J. 1980. Stomach contents analysis- a review of methods and their application. J. Fish Biol. 17: 411-429.

Keen, M.A. 1971. Sea shell of tropical west America marine mollusks from Baja California to Perú. Universidad de Stanford, California, EEUU.

Kessler, W.S. 2006. The Circulation of the eastern tropical Pacific: A revieu. Prog. Ocean. 69: 181-217.

Krebs, C.J. 1999. Ecological methodology. Addison-Wesley, California, EEUU.

Laptikhovsky, V.V. 2005. A trophic ecology of two grenadier species (Macruridae, Pisces) in deep waters of the Southwest Atlantic. Deep Sea Res. 52: 1502 1514 .

López-Peralta, R.H. \& C. Arcila. 2002. Diet composition of fish species from the southern continental shelf of Colombia. NAGA 25: 23-29.

López, P., J. Rosas, A. Velásquez, T. Cabrera \& C. Maneiro. 2002. Desarrollo embrionario y larval del bolo Diplectrum radiale Quoy y Gaimard, 1824 (Pisces: Serranidae). Biol. Mar. Ocean. 37: 127-137. 
Obando, E. \& J. León. 1989. Reproducción del bolo, Diplectrum formosum (Linnaeus, 1766) (Pisces: Serranidae) en Punta Mosquito, Isla de Margarita, Venezuela. Scie. Mar. 53: 771-777.

Pacheco, S.P. 1991. Pacifico tropical mexicano. Oceanografía física. Oceanografía de mares mexicanos. AGT, México D.F, México.

Pianka, E.R. 1973. The structure of lizard communities. Ann. Rev. Ecol. Syst. 4: 53-74.

Pinkas, L., M.S. Oliphant \& Z.L. Iverson. 1971. Food habit of albacore bluefin, tuna and bonito in California water. California Department of Fishing Game. Fish Bull. 152: 1105

Platell, M.E. \& I.C. Potter. 2001. Partitioning of food resources amongst 18 abundant benthic carnivorous fish species in marine waters on the lower west coast of Australia. J. Exp. Mar. Biol. Ecol. 261: 31-54.
Raborn, S.W., L.E. Miranda \& M.T. Driscoll. 2004. Diet overlap and consumption patterns suggest seasonal flux in the likelihood for exploitative competition among piscivorous fishes. Ecol. Fresh. Fish. 13: 276-284.

Ruíz-Ramírez, S., G. Lucano-Ramírez \& J. MariscalRomero. 1997. Length-weight relationships of softbottom demersal fishes form Jalisco and Colima states, Mexico. Naga ICLARM Q. 20: 62-63.

Salazar-Vallejo, S.I., J.A. De León-González \& H. Salaices-Polanco. 1988. Poliquetos (Anellida: Polychaeta) de México. Universidad Autónoma de Baja California Sur, La Paz, México.

Touart, L.W. \& S.A. Bortone. 2006. The accessory reproductive structure in the simultaneous hermaphrodite Diplectrum bivittatum. J. Fish Biol. 16: 397-403.

Winemiller, K.O. \& E.R. Pianka. 1990. Organization in natural assemblages of desert lizards and tropical fishes. Ecol. Monog. 60: 27-55. 